\title{
Finite size scaling of conformal theories in the presence of a near-marginal operator
}

\author{
Anqi Cheng, Anna Hasenfratz, Yuzhi Liu, Gregory Petropoulos, and David Schaich* \\ Department of Physics, University of Colorado, Boulder, CO 80309, USA
}

\begin{abstract}
The slowly evolving gauge coupling of gauge-fermion systems near the conformal window makes numerical investigations of these models challenging. We consider finite size scaling and show that this often used technique leads to inconsistent results if the leading order scaling corrections are neglected. When the corrections are included the results become consistent not only between different operators but even when data obtained at different gauge couplings or with different lattice actions are combined. Our results indicate that the $\mathrm{SU}(3)$ 12-fermion system is conformal with mass anomalous dimension $\gamma_{m}=0.235(15)$.
\end{abstract}

Strongly coupled gauge-fermion systems near the conformal window are candidates to describe the dynamics of electroweak symmetry breaking and beyond-Standard Model physics. These models are expected to have a "walking" gauge coupling and large anomalous mass dimension that can give rise to an enhanced fermion condensate, while the weakly broken conformal symmetry could lead to a light dilaton that plays the role of the Higgs boson [1]. While the non-perturbative properties of these systems are well suited to lattice studies, standard lattice methods are frequently not efficient to investigate the infrared properties of near-conformal systems. The problems are mainly due to the nearly marginal walking nature of the irrelevant gauge coupling. In this paper we investigate finite size scaling (FSS), a well established method to predict critical scaling exponents, and show that it is essential to take into account the effect of the nearly marginal coupling in correction to scaling to obtain consistent results.

We concentrate on the $\mathrm{SU}(3)$ gauge model with 12 fundamental fermions, a controversial system. Several groups have studied the infrared properties of this model using different methods and different lattice actions, arriving at contradictory conclusions regarding its infrared dynamics. (For a limited set of references see Refs. 216.) In particular FSS was considered in Refs. [46. 11, 12. Inconsistencies of the scaling exponent as predicted by different operators lead some authors to strongly question the conformal behavior of this model.

We investigate this system at many gauge coupling values, and also analyze the published meson spectrum data of the Lattice Higgs (LH) and LatKMI collaborations [4, 12. We develop a simple formalism that takes into account the effect of the leading irrelevant coupling and find consistent FSS for several operators. The conclusion is further strengthened when we combine several gauge couplings, even different lattice actions together. These results suggest conformal infrared dynamics reinforcing the interpretation suggested by our earlier studies of the bare step scaling function [7, phase transitions [17. and Dirac eigenvalues [9]. Preliminary results of our investigations have been reported in Ref. [18].

\section{FINITE SIZE SCALING}

Finite size scaling is a well understood technique to investigate the critical properties of systems governed by one relevant operator. Its derivation is easiest using renormalization group analysis and has been reviewed recently in connection with infrared conformal systems [19, 20]. Corrections to scaling due to the leading irrelevant operator have been successfully incorporated in three dimensional spin model studies, for example in Refs. 21, 22 that considered FSS of Ising model like systems on the critical surface. A recent publication [23] investigated corrections to scaling in mass deformed conformal systems in infinite volume. In this work we consider FSS of mass deformed conformal systems including leading corrections.

For concreteness we consider a system with one relevant operator $m$ with scaling dimension $y_{m}>0$. All other operators, denoted by $g_{i}$, are irrelevant with scaling exponents $y_{i}<0$. Renormalization group arguments predict that in a finite spatial volume $L^{3}$, any physical quantity " $M_{H}$ " with mass (engineering) dimension $\left[M_{H}\right]=1$ depends only on specific combinations of the couplings, and can be written as

$$
M_{H}=L^{-1} f\left(x, g_{i} m^{-y_{i} / y_{m}}\right),
$$

where $x \equiv L m^{1 / y_{m}}$. In the critical $m \rightarrow 0$ limit, $g_{i} m^{-y_{i} / y_{m}} \rightarrow 0$ and we find the familiar FSS formula

$$
M_{H}=L^{-1} f_{H}(x),
$$

where $f_{H}(x)$ is an arbitrary but unique scaling function that depends on the observable $M_{H}$. The exponent $y_{m}$ is universal and characteristic of the corresponding fixed point.

If one of the irrelevant operators, let's say $g_{0}$, is nearly marginal with scaling exponent $y_{0} \lesssim 0$, the term $g_{0} m^{-y_{0} / y_{m}}$ can remain significant and has to be included in the scaling analysis. This leads to the modified FSS formula

$$
M_{H}=L^{-1} f_{H}\left(x, g_{0} m^{\omega}\right),
$$



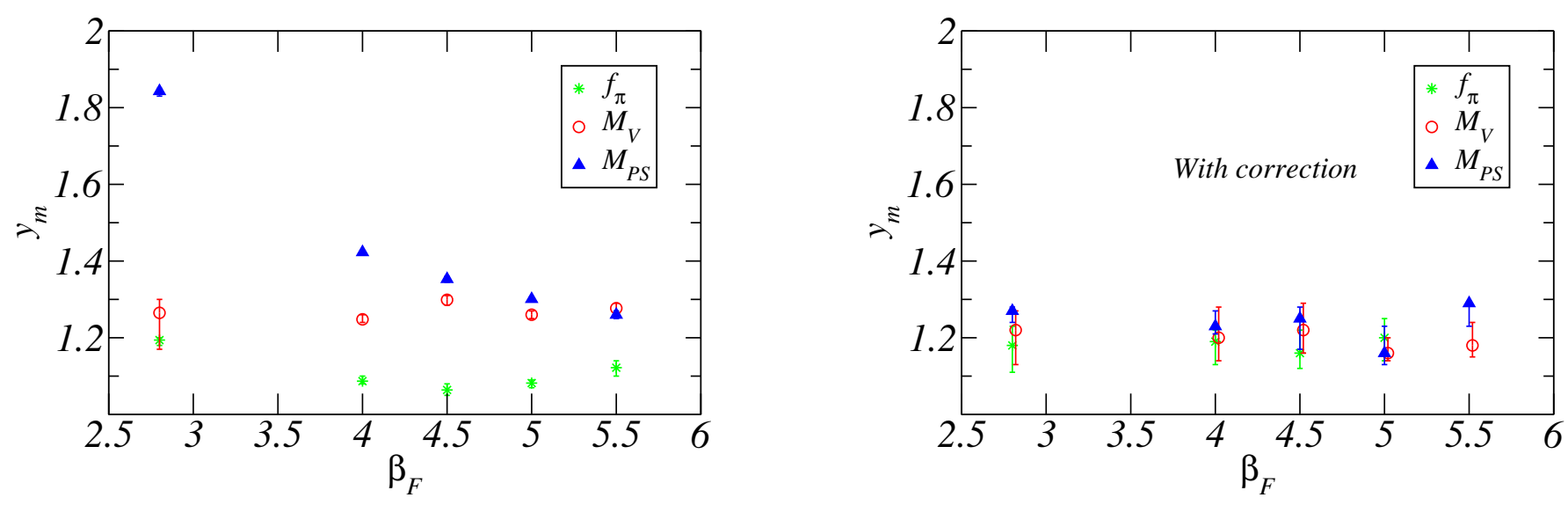

FIG. 1. The scaling dimension $y_{m}$ predicted by FSS, as a function of the gauge coupling $\beta_{F}$ for the $M_{P S}$ (blue triangles), $M_{V}$ (red circles) and $f_{\pi}$ (green $\left.\times \mathrm{s}\right)$. Left: fits including only the relevant mass operator (Eq. 2). Right: fits including both the relevant operator and leading irrelevant corrections (Eq. 5) with $y_{0}=-0.36$ fixed at the two-loop value.

where $\omega \equiv-y_{0} / y_{m} \gtrsim 0$. The scaling function $f_{H}\left(x, g_{0} m^{\omega}\right)$ is analytic even at the fixed point, and can be expanded as

$$
L M_{H}=F_{H}(x)\left\{1+g_{0} m^{\omega} G_{H}(x)+\mathcal{O}\left(g_{0}^{2} m^{2 \omega}\right)\right\} .
$$

The first term is the usual FSS expression while the second accounts for the leading corrections to scaling.

In the limit $x \rightarrow 0$, both $F_{H}(x)$ and $G_{H}(x)$ approach finite constants. In the infinite-volume limit, with small but fixed $m, F_{H}(x) \propto x$ while $G_{H}(x)$ remains finite. Our simulations cover a limited range $0.5 \lesssim x \lesssim 3$, over which we approximate $G_{H}(x)$ by a constant, $G_{H}(x)=c_{G}$, so

$$
\frac{L M_{H}}{1+c_{G} g_{0} m^{\omega}}=F_{H}(x) \text {. }
$$

One can test the validity of this approximation by using only subsets of the data restricted to smaller ranges in $x$. Equation 5 is very similar to the original Eq. 2. however, the analysis now involves three parameters: $c_{0} \equiv c_{G} g_{0}$, $y_{0}$ and $y_{m}$.

\section{FINITE SIZE SCALING FITS}

In our numerical studies we use nHYP smeared staggered fermions and a gauge action that combines fundamental and adjoint plaquette terms with $\beta_{A} / \beta_{F}=-0.25$. In Ref. 8 we reported on the phase structure and other properties of this action with $N_{f}=12$ fundamental fermions.

In the present work we consider gauge couplings $\beta_{F}=$ $2.8,4.0,4.5$ and 5.0 and volumes $12^{3} \times 24,16^{3} \times 32,20^{3} \times 40$, $24^{3} \times 48$ and $32^{3} \times 64$. The bare mass varies in the range $0.005 \leq m \leq 0.12$, such that the vector meson mass $a M_{V}<0.7$.

In the FSS analysis we approximate $F_{H}(x)$ with two independent quadratic polynomials, one at $x<x_{0}$ and the other at $x>x_{0}$. We minimize the $\chi^{2}$ of this fit in terms of the polynomial coefficients, $x_{0}, y_{m}, y_{0}$ and $c_{0}$ using a Bayesian fitter based on [24, 25] 26]. Priors on the values are $0.1 \pm 20$ for polynomial coefficients, $0.5 \pm 20$ for $-y_{0}, 1.4 \pm 1$ for $y_{m}$, and $-0.1 \pm 5$ for $c_{0}$. Table I collects the results of several different fits, listing the relevant fit parameters as well as $\chi^{2}$ per degrees of freedom (dof). This $\chi^{2}$ represents not only the goodness of the FSS "curve collapse" but the correctness of our rather simple fitting form for $F_{H}(x)$. While the latter could be improved by using a more elaborate fit function, we found the two independent quadratic polynomials to be sufficient.

Two loop perturbation theory predicts that the 12 flavor system is conformal with scaling exponent $y_{m} \approx 1.45$ and leading irrelevant exponent $y_{0} \approx-0.36$. First we analyze the data using the usual form of Eq. 2, ignoring corrections to scaling. We consider each operator and $\beta_{F}$ data set independently. The first row of Table $\mathrm{I}$ shows the result of the fit for the pseudoscalar mass $M_{P S}$ at $\beta_{F}=4.0$. This gauge coupling matches rather closely the published $\beta=2.2$ data of the LH collaboration and our prediction for $y_{m}$ is consistent with Ref. [4].

The left panel of Fig. 1 shows the results of similar analysis for the scaling exponent $y_{m}$ at other $\beta_{F}$ values for the pseudoscalar $M_{P S}$ and vector meson $M_{V}$ masses and $f_{\pi}$. The scaling exponent shows significant variations between the three observables and as the function of $\beta_{F}$, suggesting that there is no consistent FSS when using the form of Eq. 2.

When we take into account the leading scaling corrections according to Eq. 5 the situation changes. We are not able to constrain the exponent $y_{0}$ using individual data sets so at this stage we fix $y_{0}=-0.36$, the perturbative 2-loop value. The correction term decreases $\chi^{2}$ by more than a factor of two as the second row of Table I shows. We obtain consistent results when fitting only the 


\begin{tabular}{|c|c|c|c|c|c|c|}
\hline Op. & $\beta$ & $y_{m}$ & $y_{0}$ & $c_{0}(\mathrm{PS})$ & $s_{m}$ & $\chi^{2}[\mathrm{dof}]$ \\
\hline \hline PS & 4.0 & $1.421(3)$ & - & 0 & - & $3.3[35]$ \\
\hline PS & 4.0 & $1.223(17)$ & $-0.36($ fixed $)$ & $-0.66(5)$ & - & $1.3[36]$ \\
\hline PS & 4.0 & $1.228(16)$ & $-0.499(58)$ & $-0.70(6)$ & 1 & $1.1[58]$ \\
& 4.5 & & & $-0.50(6)$ & 0.73 & \\
\hline PS & 2.8 & $1.248(13)$ & $-0.466(16)$ & $-1.27(2)$ & 3.03 & $2.9[99]$ \\
& 4.0 & & & $-0.60(4)$ & 1 & \\
& 4.5 & & & $-0.40(5)$ & 0.73 & \\
& 5.0 & & & $-0.33(5)$ & 0.58 & \\
\hline PS & 4.0 & $1.238(13)$ & $-0.508(55)$ & $-0.67(5)$ & 1 & $1.4[95]$ \\
& 4.5 & & & $-0.46(5)$ & 0.73 & \\
& LH & & & $-0.82(6)$ & 1.11 & \\
& KMI 3.7 & & & $-0.76(6)$ & 0.64 & \\
& KMI 4.0 & & & $-0.70(5)$ & 0.55 & \\
\hline PS, & 2.8 & $1.228(11)$ & $-0.446(14)$ & $-1.28(2)$ & 3.03 & $2.4[191]$ \\
V & 4.0 & & & $-0.66(3)$ & 1 & \\
& 4.5 & & & $-0.48(4)$ & 0.73 & \\
& 5.0 & & & $-0.41(4)$ & 0.58 & \\
\hline PS, & 2.8 & $1.241(11)$ & $-0.465(14)$ & $-1.28(2)$ & 3.03 & $3.0[283]$ \\
V, & 4.0 & & & $-0.62(3)$ & 1 & \\
$f_{\pi}$ & 4.5 & & & $-0.43(4)$ & 0.73 & \\
& 5.0 & & & $-0.36(4)$ & 0.58 & \\
\hline
\end{tabular}

TABLE I. Results of the FSS analysis in the 12 flavor system. $M_{P S}, M_{V}$ and $f_{\pi}$ are analyzed at various $\beta_{F}$ couplings with the nHYP action, combined with the published data of the LH and LatKMI collaborations [4, 12]. $c_{0}$ denotes the amplitude of the leading correction (given only for the pseudoscalar) and $s_{m}$ is the matching scale factor of the bare mass relative to the $\beta_{F}=4.0 \mathrm{nHYP}$ data. The last column lists the $\chi^{2}$ per degrees of freedom and dof of the fit.

small $(x<1.4)$ or large $(x>1.1)$ regions, justifying our approximation of constant $G(x)=c_{G}$.

Repeating this analysis at other gauge couplings leads to the results plotted on the right panel of Fig. 11, showing consistency between all three operators in the whole $\beta_{F}$ range investigated. Not surprisingly the errors are significantly larger than before, especially for $f_{\pi}$ where the data constrain the correction coefficient $c_{0}$ only weakly.

If the gauge coupling is an irrelevant operator, the scaling function $F_{H}(x)$ is independent of $\beta_{F}$ and we can significantly strengthen the FSS fit by combining data from different gauge couplings. This requires the introduction of a set of new parameters $s_{m}$ that rescale the bare mass at each gauge coupling $m \rightarrow s_{m} m$ to a common reference value. We choose $s_{m}=1$ at $\beta_{F}=4.0$. While the scale factors $s_{m}$ depend on the gauge coupling, they are independent of the operator. Such global fits allow us to determine both scaling dimensions $y_{m}$ and $y_{0}$. However higher order corrections to scaling not accounted for in Eq. 5 can be different for different data sets and significantly increase $\chi^{2}$, especially when very different couplings are combined.

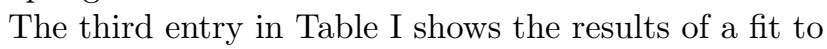
the pseudoscalar mass that combines couplings $\beta_{F}=4.0$ and 4.5. The universal fit parameters $y_{m}$ and $y_{0}$ are consistent with previous values with similar $\chi^{2} /$ dof. Includ- ing the data sets at $\beta_{F}=2.8$ and 5.0 does not change the predicted values, though we observe a significant increase in $\chi^{2}$ as the fourth entry of Table I shows. This is not surprising considering that the scale factors $s_{m}$ change by a factor of five in this case. While the scale factors increase with decreasing $\beta_{F}$, the $c_{0}$ coefficients decrease, suggesting that the conformal infrared fixed point (or its projection to the $\beta_{F}$ axis) where $c_{0}=0$ occurs at weaker gauge coupling.

We can combine different lattice actions, not only gauge couplings, in the FSS fit. Both the LH and LatKMI collaborations [4, 12] published some of their spectrum results which we can fit together with our nHYP action data. As the next entry of Table ] shows, such a combined fit for $M_{P S}$ has a small $\chi^{2} /$ dof with scaling dimensions consistent with previous fits. The left panel of Fig. 2 illustrates the "curve collapse" of this fit.

The vector meson mass can be analyzed similarly, and one can even combine it with the pseudoscalar. The fit now depends on two independent scaling functions $F_{H}(x)$ for the two operators. As the fifth entry in Table 1 shows the scaling dimensions from such a combined fit are consistent with the pseudoscalar fit with similar $\chi^{2} /$ dof. The errors listed are statistical only and do not take into account possible correlations between the two operators.

The pion decay constant is expected to scale with the same universal exponents as the hadron masses but it exhibits very different finite volume effects [4, 12] and corrections to scaling could be more significant to $f_{\pi}$ than to the masses, especially in small volumes. As the volume decreases the mesonic bound states are squeezed. At some point the volume might become too small to support bound states and the physical meaning of $f_{\pi}$ changes. The first order phase transition observed with Wilson fermions in Ref. [27] could be of similar origin. Nevertheless a combined fit for $M_{P S}, M_{V}$, and $f_{\pi}$ is reasonable even when we combine the gauge couplings $\beta_{F}=2.8,4.0,4.5$ and 5.0 as shown in the last entry of Table I. In this case we fit nearly 300 data points with three independent scaling functions and $12 c_{0}$ coefficients describing the leading corrections to scaling, yet the predicted scaling exponents are consistent with all previous fit results.

\section{CONCLUSION}

We have demonstrated that apparent inconsistencies in finite size scaling analysis of the $N_{f}=12$ system can be resolved by considering the effect of the leading irrelevant coupling, at least for the pseudoscalar and vector meson masses and $f_{\pi}$. For these quantities combined fits of several independent data sets at different gauge couplings and even different lattice actions are consistent with conformal infrared dynamics. Based on various fits presented in Table 1 we predict the anomalous mass di- 

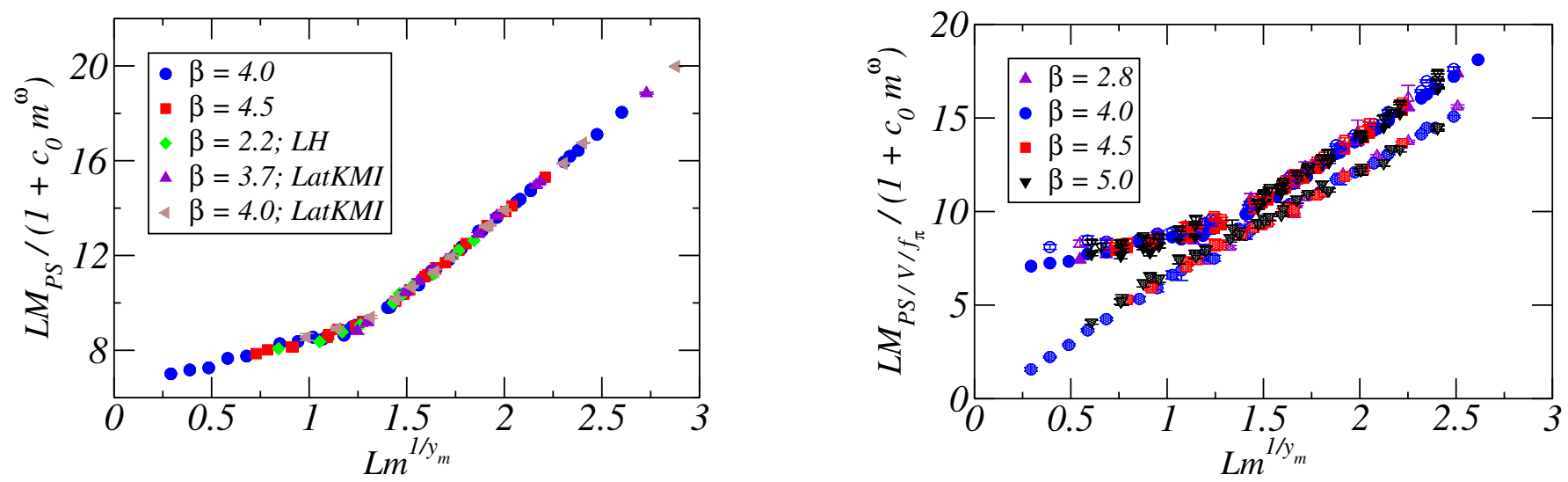

FIG. 2. Left panel: The best curve collapse fit for the $M_{P S}$ combining data at $\beta_{F}=4.0,4.5$ and the published data of the LH and LatKMI collaborations [4, 12. The fit parameters are listed in Table I] Right panel: Similar fit combining $M_{P S}$ (filled symbols), $M_{V}$ (open symbols), and $f_{\pi}$ (shaded symbols) using data at $\beta_{F}=2.8,4.0,4.5$ and 5.0. The values for $f_{\pi}$ are rescaled by a factor of 9 for better clarity.

mension $\gamma_{m}=y_{m}-1=0.235(15)$ at the corresponding infrared fixed point.

We have investigated only three physical quantities and cannot prove that all other observables will scale consistently once corrections to scaling are taken into account - especially because these corrections might be more important to some observables than to others. It will be important to consider other quantities, especially those related to the static potential as published large volume data appear to be inconsistent with conformal dynamics [10.

We expect that systems near the conformal boundary will generically possess a nearly-marginal operator due to the walking gauge coupling, or possibly even some other operator that becomes relevant at the conformal boundary. The results presented in this paper suggest that such an operator has important effects that have to be considered when studying any strongly-coupled manyflavor system.

\section{ACKNOWLEDGMENTS}

We thank Biagio Lucini for suggesting that we include corrections in the FSS analysis, Roman Zwicky for useful discussions concerning the correction terms and Julius Kuti for his helpful probing questions. Part of this work was performed when A. H. and D. S. visited the Aspen Center for Physics (NSF Grant No. 1066293) and $\mathrm{CP}^{3}$-Origins in Odense, and we thank both institutions for their support and hospitality. A. H. is grateful for the hospitality of the Brookhaven National Laboratory HET group during her extended visit. This research was partially supported by the U.S. Department of Energy (DOE) through Grant No. de-sc0010005 (A. C., A. H., Y. L., and D. S.) and by the DOE Office of Science Graduate Fellowship Program under Contract No. DE-
AC05-06OR23100 (G. P.). Our code is based in part on the MILC Collaboration's public lattice gauge theory software 28. Numerical calculations were carried out on the HEP-TH and Janus clusters, partially funded by NSF Grant No. CNS-0821794 at the University of Colorado; at Fermilab under the auspices of USQCD supported by the DOE; and at the San Diego Computing Center through XSEDE supported by National Science Foundation Grant No. OCI-1053575.

* Present address: Department of Physics, Syracuse University, Syracuse, NY 13244, USA

[1] For brief reviews, see T. Appelquist, R. Brower, S. Catterall, G. Fleming, J. Giedt, A. Hasenfratz, J. Kuti, E. Neil and D. Schaich (2013), 1309.1206, URL http: //www.usqcd.org/documents/bsm.pdf, and references therein.

[2] T. Appelquist, G. T. Fleming, and E. T. Neil, Phys. Rev., D79, 076010 (2009) arXiv:0901.3766.

[3] A. Deuzeman, M. P. Lombardo, and E. Pallante, Phys. Rev., D82, 074503 (2010), arXiv:0904.4662.

[4] Z. Fodor, K. Holland, J. Kuti, D. Nogradi, and C. Schroeder, Phys. Lett., B703, 348 (2011), arXiv:1104.3124

[5] T. Appelquist, G. T. Fleming, M. F. Lin, E. T. Neil, and D. Schaich, Phys. Rev., D84, 054501 (2011), arXiv:1106.2148

[6] T. DeGrand, Phys. Rev., D84, 116901 (2011), arXiv:1109.1237

[7] A. Hasenfratz, Phys. Rev. Lett., 108, 061601 (2012), arXiv: 1106.5293

[8] A. Cheng, A. Hasenfratz, and D. Schaich, Phys. Rev., D85, 094509 (2012), arXiv:1111.2317.

[9] A. Cheng, A. Hasenfratz, G. Petropoulos, and D. Schaich, JHEP, 1307, 061 (2013), arXiv:1301.1355.

[10] Z. Fodor, K. Holland, J. Kuti, D. Nogradi, C. Schroeder, and C. H. Wong, PoS, Lattice 2012, 025 (2012), 
arXiv: 1211.3548

[11] Z. Fodor, K. Holland, J. Kuti, D. Nogradi, C. Schroeder, and C. H. Wong, PoS, Lattice 2012, 279 (2012), arXiv: 1211.4238

[12] Y. Aoki, T. Aoyama, M. Kurachi, T. Maskawa, K.-i. Nagai, H. Ohki, A. Shibata, K. Yamawaki, and T. Yamazaki, Phys. Rev., D86, 054506 (2012), arXiv:1207.3060.

[13] Y. Aoki, T. Aoyama, M. Kurachi, T. Maskawa, K.-i. Nagai, H. Ohki, E. Rinaldi, A. Shibata, K. Yamawaki, and T. Yamazaki, (2013), arXiv:1302.4577

[14] E. Itou, PTEP, 2013, 083B01 (2013) arXiv:1212.1353.

[15] C.-J. D. Lin, K. Ogawa, H. Ohki, and E. Shintani, JHEP, 1208, 096 (2012), arXiv:1205.6076

[16] X.-Y. Jin and R. D. Mawhinney, PoS, Lattice 2011, 066 (2012), arXiv:1203.5855

[17] A. Hasenfratz, A. Cheng, G. Petropoulos, and D. Schaich, (2013), arXiv:1303.7129.

[18] A. Hasenfratz, A. Cheng, G. Petropoulos, and D. Schaich, PoS, LATTICE 2013, 075 (2013),
arXiv:1310.1124

[19] T. DeGrand and A. Hasenfratz, Phys. Rev., D80, 034506 (2009), arXiv:0906.1976

[20] L. Del Debbio and R. Zwicky, Phys. Rev., D82, 014502 (2010), arXiv:1005.2371

[21] M. Hasenbusch, J.Phys., A32, 4851 (1999), arXiv:heplat/9902026 [hep-lat]

[22] M. Hasenbusch, Phys.Rev., B82, 174433 (2010).

[23] L. Del Debbio and R. Zwicky, (2013), arXiv:1306.4038.

[24] G. Lepage, B. Clark, C. Davies, K. Hornbostel, P. Mackenzie, et al., Nucl.Phys.Proc.Suppl., 106, 12 (2002), arXiv:hep-lat/0110175 [hep-lat]

[25] K. Hornbostel, G. Lepage, C. Davies, R. Dowdall, H. Na, et al., Phys.Rev., D85, 031504 (2012), arXiv:1111.1363 [hep-lat].

[26] https://github.com/gplepage/corrfitter.

[27] K. I. Ishikawa, Y. Iwasaki, Y. Nakayama, and T. Yoshie, (2013), arXiv:1310.5049 [hep-lat]

[28] http://www.physics.utah.edu/ $\operatorname{detar/milc/.~}$ 